\title{
A new software framework for implementing crystal growth models to materials of any crystallographic complexity
}

Yongsheng Zhao, ${ }^{\dagger}$ Carl J. Tilbury, ${ }^{\dagger}$ Steven Landis,${ }^{\ddagger}$ Yuanyuan Sun, ${ }^{\dagger}$ Jinjin Li,${ }^{\S}$ Peng Zhu $,{ }^{\dagger}, \S$ and Michael F. Doherty*,†

'Department of Chemical Engineering, University of California Santa Barbara, California 93106-5080, USA

tDepartment of Electrical and Computer Engineering, University of California Santa Barbara, California 93106-9560, USA

${ }^{\S}$ National Key Laboratory of Science and Technology on Micro/Nano Fabrication, Department of Micro/Nano Electronics, Shanghai Jiao Tong University,

Shanghai 200240, China

* Corresponding author. Tel.: +1 805-893-5309

E-mail addresses: mfd@ucsb.edu 


\section{Solvent-Solute Interfacial Energy}

Accounting for the molecular interaction energetics is critical to the mechanistic model, and the presence of a solvent alters the relevant surface interactions due to solvation of the crystal surface. To correctly predict this effect one must determine how these interactions are modified by the presence of a solvent. Israelachvili (chapter 17) [1] recommends the classical interface equation proposed by Dupré in which solvent-solute interfacial energies are estimated as a sum of the cohesive energy of each phase $\left(\gamma_{X}\right.$ and $\left.\gamma_{S}\right)$ minus the work of adhesion $\left(W_{a d, X S}\right)$.

$$
\gamma_{X S}=\gamma_{X}+\gamma_{S}-W_{a d, X S}
$$

where $X$ is crystal, and $S$ is solvent.

There are two different approaches to calculating the solvent-modified crystal bond energy.

(1) Sum then modify approach. Sum up all the solid-solid interactions that contribute to the total bond energy, then solvent-modify that total bond.

(2) Modify then sum approach. Solvent-modify each individual interaction and then sum up all the solvent-modified bonds to obtain the total solvent-modified bond energy.

In general, these approaches lead to a different end value.

\section{vOCG Solvent Model}

Based on the van Oss, Chaudhury and Good (vOCG) model [2-4], Eq. (1) can be rewritten as follows: 


$$
\begin{aligned}
\gamma_{X S} & =\gamma_{X}+\gamma_{S}-W_{a d, X S} \\
& =\left(\gamma_{X}^{d}+\gamma_{X}^{A B}\right)+\left(\gamma_{S}^{d}+\gamma_{S}^{A B}\right)-\left(W_{a d, X S}^{d}+W_{a d, X S}^{A B}\right) \\
& =\left(\gamma_{X}^{d}+\gamma_{X}^{A B}\right)+\left(\gamma_{S}^{d}+\gamma_{S}^{A B}\right)-\left(2 \sqrt{\gamma_{X}^{d} \gamma_{S}^{d}}+W_{a d, X S}^{A B}\right) \\
& =\left(\gamma_{X}^{d}+\gamma_{X}^{A B}\right)+\left(\gamma_{S}^{d}+\gamma_{S}^{A B}\right)-\left(2 \sqrt{\gamma_{X}^{d} \gamma_{S}^{d}}+2 \sqrt{\gamma_{X}^{+} \gamma_{S}^{-}}+2 \sqrt{\gamma_{X}^{-} \gamma_{S}^{+}}\right)
\end{aligned}
$$

where $d$ is dispersive interaction, $A B$ is acid-base interaction, and the superscripts + and represent electron-accepting and electron-donating components of crystal and solvent, respectively.

For the solvent side, the dispersive energy $\gamma_{S}^{d}$ is calculated using Eq. (3). The solvent acid-base energy $\gamma_{S}^{A B}$ (Eq. (4)) is calculated by subtracting $\gamma_{S}^{d}$ from the solvent total surface energy $\gamma_{S}$ in the form of Eq. (5) (for alcohols), Eq. (6) (for acids, phenols, amines and water), and Eq. (7) (for all other solvents), respectively [5].

$$
\begin{aligned}
& \gamma_{S}^{d}=0.0715\left(V_{m}\right)^{1 / 3}\left(\delta_{d}\right)^{2} \\
& \gamma_{S}^{A B}=\gamma_{S}-\gamma_{S}^{d} \\
& \gamma_{S}=0.0715\left(V_{m}\right)^{1 / 3}\left(\delta_{d}^{2}+\delta_{p o l}^{2}+0.06 \delta_{h b}^{2}\right) \\
& \gamma_{S}=0.0715\left(V_{m}\right)^{1 / 3}\left(\delta_{d}^{2}+2 \delta_{p o l}^{2}+0.481 \delta_{h b}^{2}\right) \\
& \gamma_{S}=0.0715\left(V_{m}\right)^{1 / 3}\left(\delta_{d}^{2}+0.632 \delta_{p o l}^{2}+0.632 \delta_{h b}^{2}\right)
\end{aligned}
$$

where $V_{m}$ is the molar volume of the solvent, $\delta_{d}$ is the dispersive solubility parameter, $\delta_{p o l}$ is the polarization solubility parameter, and $\delta_{h b}$ is the hydrogen bonding solubility parameter, respectively. These $\delta$ values are tabulated in reference [6], and are stored in the ADDICT3 database.

For the crystal side, we use the sum then modify approach. The following equations are used: 


$$
\begin{aligned}
& \gamma_{X}^{d}=\frac{E_{P B C}^{d}}{S A} \\
& \gamma_{X}^{A B}=\frac{E_{P B C}^{A B}}{S A}
\end{aligned}
$$

where $E_{P B C}^{d}$ is the dispersive term of the full $\mathrm{PBC}\left(E_{P B C}\right)$ interaction strengths (actually it is the total van der Waals term), $E_{P B C}^{A B}$ is the coulombic term of the full PBC interaction strengths, and the surface area $(S A)$ is $2\left(V_{m}\right)^{2 / 3}$, since 2 surfaces are formed.

In ADDICT3's Hydrogen_bond.m program, we determine whether there are hydrogen bonds between a given growth unit and its neighboring growth units along the chain, based on the type of atoms, the distance between atoms, and the interaction energies. Based on these parameters, we determine which type of hydrogen bond exists between the growth units. The types are: medium H-bond (class 2), weak H-bond (class 1), no H-bond (class 0). For example, in Figure S1 consider growth unit 1 (gu1) as the given growth unit. It has 3 bonds in the reverse chain direction, which are bond 1 (black solid line), bond 2 (blue dotted line), and bond 3 (purple dotted line) in the figure. The total energy $E_{P B C, g u 1}^{T o t a l}$ between the growth unit 1 and its neighboring growth units in the reverse chain direction can be calculated by Eq. (10), and the acid-base energy $E_{P B C, g u 1}^{A B}$ is equal to the sum of the respective coulombic terms of bonds 1-3 as shown in Eq. (11). There are two cases of H-bond crystals that lead to two different ways of evaluating the terms in Eq. (11). In the first case, when there is a medium H-bond between gu1 and a neighbor along the chain (e.g., gu2), acid-base energy of the class $2 \mathrm{H}$-bond is equal to the coulombic term of the class 2 bond, and the acid-base energy of class 1 and class 0 bonds are set equal to 0 . Basically we can set the coulombic term of other bonds 
to zero if we have a reasonably strong $\mathrm{H}$ bond, since that will have a large $\mathrm{AB}$ term. The second case is when there is no medium H-bond; now the acid-base energy for each bond in Eq. (11) is equal to the coulombic term of class 1 or class 0.

$$
\begin{aligned}
E_{P B C, g u 1}^{T o t a l}= & E_{P B C, g u 1}^{A B}+E_{P B C, g u 1}^{d} \\
= & E_{P B C, \text { bond } 1}^{\text {total }}+E_{P B C, \text { bond } 2}^{\text {total }}+E_{P B C, \text { bond } 3}^{\text {total }} \\
= & E_{P B C, \text { bond } 1}^{A B}+E_{P B C, \text { bond } 2}^{A B}+E_{P B C, \text { bond } 3}^{A B} \\
& +E_{P B C, \text { bond } 1}^{d}+E_{P B C, \text { bond } 2}^{d}+E_{P B C, \text { bond } 3}^{d}
\end{aligned}
$$

For example, when bond 1 belongs to class 2, bond 2 belongs to class 1, and bond 3 belongs to class 0 , the acid-base energy of bond 1 is equal to its coulombic term, and the acid-base of bond 2 and bond 3 is equal to 0 as shown in Eq. (12). However, when bond 1 belongs to class 1 , bond 2 belongs to class 1 , and bond 3 belongs to class 0 , the acid-base energy of the three bonds is equal to their respective coulombic term as shown in Eq. (11).

$$
\begin{aligned}
& E_{P B C, g u 1}^{A B}=E_{P B C, b \text { ond } 1}^{A B}+E_{P B C, b \text { ond } 2}^{A B}+E_{P B C, \text { bond } 3}^{A B} \\
& E_{P B C, g u 1}^{A B}=E_{P B C, \text { bond } 1}^{A B}+0+0
\end{aligned}
$$

chain direction

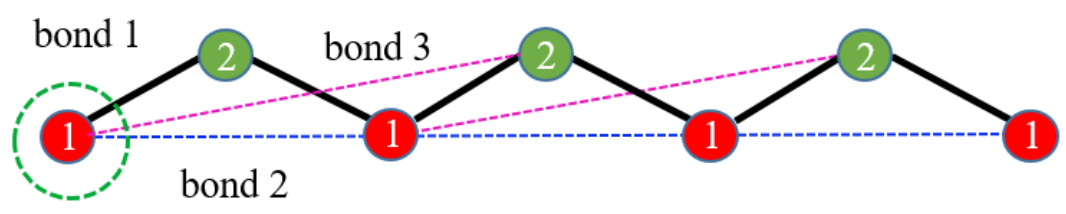

Figure S1. A PBC consisting of black bonds and two growth units (1 and 2). The growth unit 1 in the green dotted circle has three bonds in the reverse chain direction (black, blue, and purple bonds).

For the work of adhesion [2-5], the following equations are employed: 


$$
\begin{aligned}
& \gamma_{X}^{+}=\gamma_{X}^{-}=\frac{\gamma_{X}^{A B}}{2} \\
& \gamma_{S}^{-}=\frac{\gamma_{S}^{A B}}{2 \sqrt{\text { ratio }_{S}}} \\
& \gamma_{S}^{+}=\gamma_{S}^{-} \times \text {ratio }_{S} \\
& \text { ratio }_{S}=\frac{\gamma_{S}^{+}}{\gamma_{S}^{-}}
\end{aligned}
$$

where $\gamma_{X}^{A B}$ is known from Eq. (9), and $\gamma_{S}^{A B}$ is known from Eq. (4). Therefore, in order to obtain the energy values in Eqs (13)-(14), it is necessary to obtain the ratio value of the solvent $\left(\right.$ ratio $\left._{S}\right)$. For different solvents, the ratio $_{S}$ values are $1,0.1$ or 4.75 , respectively (the specific ratio $_{S}$ values for different solvents are tabulated in the ADDICT3 database; the value 4.75 is for water only; the other two values, 1 and 0.1 are for various organic solvents).

\section{Kaelble Solvent Model}

For the solvent effect method of our previous version of ADDICT (ADDICT2), the solvent-solute interfacial energy decomposition is the same as the vOCG model (Eqs (1)-(2)). However, for the solvent side, $\gamma_{S}^{d}$ and $\gamma_{S}^{A B}$ are calculated using the following equations:

$$
\begin{aligned}
& \gamma_{S}^{d}=f \times\left(V_{m}\right)^{1 / 3}\left(\delta_{d}\right)^{2} \\
& \gamma_{S}^{A B}=f \times\left(V_{m}\right)^{1 / 3}\left(\delta_{A B}\right)^{2} \\
& \gamma_{S}=f \times\left(V_{m}\right)^{1 / 3}\left(\delta_{\text {total }}\right)^{2} \\
& \delta_{A B}^{2}=\delta_{p o l}^{2}+\delta_{h b}^{2}
\end{aligned}
$$


where $f$ is dimensionless scaling parameter, $\delta_{A B}$ is the acid-base solvent solubility parameter, and other parameters in the above equation have the same meaning as the parameters in Eqs (3)-(7).

For the crystal side, we use the modify then sum approach. The quantities $\gamma_{X}^{d}$ and $\gamma_{X}^{A B}$ are calculated based on the following equations:

$$
\begin{aligned}
& \gamma_{X}^{d}=\frac{\frac{1}{2} E_{P B C}^{d}}{S} \\
& \gamma_{X}^{A B}=\frac{\frac{1}{2} E_{P B C}^{A B}}{S}
\end{aligned}
$$

where the $\gamma_{X}^{d}, \gamma_{X}^{A B}, E_{P B C}^{d}$, and $E_{P B C}^{A B}$ are consistent with the meaning of parameters in Eqs (8)-(9). It should be noted that the dispersive and coulombic terms together are half of the corresponding PBC energy. The surface area $(S)$ is $\left(V_{m}\right)^{2 / 3}$.

When the electrostatic interaction between two growth units is greater than 0.2 times the total interaction $\left(\gamma_{X}^{A B}>0.2 \gamma_{X}\right)$, we assume that there is a hydrogen bond. When there is a hydrogen bond, all calculations remain the same. When there is no hydrogen bond, we don't want to model the electrostatic interaction as if it were there because the electrostatic interaction value is relatively small. Therefore, $\gamma_{X}^{A B}$ is set to 0 and $\gamma_{X}^{d}$ is set equal to $\gamma_{X}$.

For the work of adhesion [2-5], the following equations are used

$$
\begin{gathered}
\gamma_{X}^{+}=\gamma_{X}^{-}=\frac{\gamma_{X}^{A B}}{2} \\
\gamma_{S}^{+}=\gamma_{S}^{-}=\frac{\gamma_{S}^{A B}}{2}
\end{gathered}
$$


where $\gamma_{S}^{A B}$ is known from Eq. (18), and $\gamma_{X}^{A B}$ is known from Eq. (22). As can be seen from the above equations, the ratio $\gamma_{S}^{+} / \gamma_{S}^{-}=1$ in the Kaelble solvent model.

\section{References}

[1] Israelachvili, J. N. (2011). Intermolecular and Surface Forces. Academic press, Third Edition.

[2] van Oss, C. J., Good, R. J., \& Chaudhury, M. K. (1988). Additive and nonadditive surface tension components and the interpretation of contact angles. Langmuir, 4(4), 884-891.

[3] van Oss, C. J., Chaudhury, M. K., \& Good, R. J. (1987). Monopolar surfaces. Advances in colloid and interface science, 28, 35-64.

[4] van Oss, C. J., Chaudhury, M. K., \& Good, R. J. (1988). Interfacial Lifshitz-van der Waals and polar interactions in macroscopic systems. Chemical Reviews, 88(6), 927-941.

[5] Tilbury, C. J., Green, D. A., Marshall, W. J., \& Doherty, M. F. (2016). Predicting the effect of solvent on the crystal habit of small organic molecules. Crystal Growth \& Design, 16(5), 2590-2604.

[6] Hansen, C. M. (2002). Hansen solubility parameters: a user's handbook. CRC press. 
Table S1: Detailed Results: naphthalene grown from ethanol using the vOCG solvent model*

\begin{tabular}{|c|c|c|c|c|c|c|c|c|}
\hline Face & Step edge & $\Phi_{P B C}$ & $a_{P}$ & $a_{E}$ & $l_{c}$ & Step velocity & $d_{h k l}$ & $R$ \\
\hline \multirow[t]{6}{*}{$\left(\begin{array}{lll}0 & 0 & 1\end{array}\right)$} & {$\left[\begin{array}{lll}0 & -1 & 0\end{array}\right]$} & 0.33 & 4.11 & 5.97 & 169.00 & 4.77 & 7.24 & 1 \\
\hline & {$\left[\begin{array}{lll}-1 & -1 & 0\end{array}\right]$} & 0.49 & 4.83 & 5.08 & 212.44 & 4.87 & & \\
\hline & {$\left[\begin{array}{lll}-1 & 1 & 0\end{array}\right]$} & 0.49 & 4.83 & 5.08 & 212.44 & 4.87 & & \\
\hline & {$\left[\begin{array}{lll}0 & 1 & 0\end{array}\right]$} & 0.33 & 4.11 & 5.97 & 169.00 & 4.77 & & \\
\hline & {$\left[\begin{array}{lll}1 & 1 & 0\end{array}\right]$} & 0.49 & 4.83 & 5.08 & 212.44 & 4.87 & & \\
\hline & {$\left[\begin{array}{lll}1 & -1 & 0\end{array}\right]$} & 0.49 & 4.83 & 5.08 & 212.44 & 4.87 & & \\
\hline \multirow[t]{4}{*}{$\left(\begin{array}{lll}1 & 1 & 0\end{array}\right)$} & {$\left[\begin{array}{lll}-1 & 1 & 0\end{array}\right]$} & 0.43 & 7.77 & 5.08 & 189.54 & 8.23 & 4.50 & 1.49 \\
\hline & {$\left[\begin{array}{lll}-1 & 1 & -2\end{array}\right]$} & 0.19 & 5.02 & 7.86 & 125.61 & 6.48 & & \\
\hline & {$\left[\begin{array}{lll}1 & -1 & 0\end{array}\right]$} & 0.43 & 7.77 & 5.08 & 189.54 & 8.23 & & \\
\hline & {$\left[\begin{array}{lll}1 & -1 & 2\end{array}\right]$} & 0.19 & 5.02 & 7.86 & 125.61 & 6.48 & & \\
\hline \multirow[t]{6}{*}{$\left(\begin{array}{lll}2 & 0 & -1\end{array}\right)$} & {$\left[\begin{array}{lll}0 & -1 & 0\end{array}\right]$} & 0.30 & 7.27 & 5.97 & 155.57 & 8.63 & 4.09 & 1.31 \\
\hline & {$\left[\begin{array}{lll}1 & -1 & 2\end{array}\right]$} & 0.18 & 5.53 & 7.86 & 123.18 & 7.16 & & \\
\hline & {$\left[\begin{array}{lll}1 & 1 & 2\end{array}\right]$} & 0.18 & 5.53 & 7.86 & 123.18 & 7.16 & & \\
\hline & {$\left[\begin{array}{lll}0 & 1 & 0\end{array}\right]$} & 0.30 & 7.27 & 5.97 & 155.57 & 8.63 & & \\
\hline & {$\left[\begin{array}{lll}-1 & 1 & -2\end{array}\right]$} & 0.18 & 5.53 & 7.86 & 123.18 & 7.16 & & \\
\hline & {$\left[\begin{array}{lll}-1 & -1 & -2\end{array}\right]$} & 0.18 & 5.53 & 7.86 & 123.18 & 7.16 & & \\
\hline \multirow[t]{4}{*}{$\left(\begin{array}{lll}1 & 1 & -1\end{array}\right)$} & {$\left[\begin{array}{lll}-1 & 1 & 0\end{array}\right]$} & 0.41 & 7.52 & 5.08 & 181.20 & 8.11 & 4.65 & 1.61 \\
\hline & {$\left[\begin{array}{lll}-1 & -1 & -2\end{array}\right]$} & 0.18 & 4.86 & 7.86 & 123.75 & 6.29 & & \\
\hline & {$\left[\begin{array}{lll}1 & -1 & 0\end{array}\right]$} & 0.41 & 7.52 & 5.08 & 181.20 & 8.11 & & \\
\hline & {$\left[\begin{array}{lll}1 & 1 & 2\end{array}\right]$} & 0.18 & 4.86 & 7.86 & 123.75 & 6.29 & & \\
\hline
\end{tabular}

${ }^{*} \Phi_{P B C}$ is the kink energy in $\mathrm{kcal} / \mathrm{mol} ; a_{P}$ is the propagation length in $\AA$; $a_{E}$ is the growth unit width along each chain in $\AA ; l_{c}$ is the critical length in $\AA ; R$ is the relative growth rate of each face; $S=1.04$ and $T=298 \mathrm{~K}$. 
Table S2: Detailed Results: naphthalene grown from cyclohexane using the vOCG solvent model*

\begin{tabular}{|c|c|c|c|c|c|c|c|c|}
\hline Face & Step edge & $\Phi_{P B C}$ & $a_{P}$ & $a_{E}$ & $l_{c}$ & Step velocity & $d_{h k l}$ & $R$ \\
\hline \multirow[t]{6}{*}{$\left(\begin{array}{lll}0 & 0 & 1\end{array}\right)$} & {$\left[\begin{array}{lll}0 & -1 & 0\end{array}\right]$} & 0.36 & 4.11 & 5.97 & 720.31 & 8.23 & 7.24 & 1 \\
\hline & {$\left[\begin{array}{lll}-1 & -1 & 0\end{array}\right]$} & 0.30 & 4.83 & 5.08 & 523.14 & 8.59 & & \\
\hline & {$\left[\begin{array}{lll}-1 & 1 & 0\end{array}\right]$} & 0.30 & 4.83 & 5.08 & 523.18 & 8.58 & & \\
\hline & {$\left[\begin{array}{lll}0 & 1 & 0\end{array}\right]$} & 0.36 & 4.11 & 5.97 & 720.31 & 8.23 & & \\
\hline & {$\left[\begin{array}{lll}1 & 1 & 0\end{array}\right]$} & 0.30 & 4.83 & 5.08 & 523.14 & 8.59 & & \\
\hline & {$\left[\begin{array}{lll}1 & -1 & 0\end{array}\right]$} & 0.30 & 4.83 & 5.08 & 523.18 & 8.58 & & \\
\hline \multirow[t]{4}{*}{$\left(\begin{array}{lll}1 & 1 & 0\end{array}\right)$} & {$\left[\begin{array}{lll}-1 & 1 & 0\end{array}\right]$} & 0.26 & 7.77 & 5.08 & 448.69 & 14.28 & 4.50 & 3.88 \\
\hline & {$\left[\begin{array}{lll}-1 & 1 & -2\end{array}\right]$} & 0.12 & 5.02 & 7.86 & 312.10 & 10.17 & & \\
\hline & {$\left[\begin{array}{lll}1 & -1 & 0\end{array}\right]$} & 0.26 & 7.77 & 5.08 & 448.69 & 14.28 & & \\
\hline & {$\left[\begin{array}{lll}1 & -1 & 2\end{array}\right]$} & 0.12 & 5.02 & 7.86 & 312.10 & 10.17 & & \\
\hline \multirow[t]{6}{*}{$\left(\begin{array}{lll}2 & 0 & -1\end{array}\right)$} & {$\left[\begin{array}{lll}0 & -1 & 0\end{array}\right]$} & 0.31 & 7.27 & 5.97 & 636.46 & 15.19 & 4.09 & 1.12 \\
\hline & {$\left[\begin{array}{lll}1 & -1 & 2\end{array}\right]$} & 0.11 & 5.53 & 7.86 & 296.52 & 11.24 & & \\
\hline & {$\left[\begin{array}{lll}1 & 1 & 2\end{array}\right]$} & 0.11 & 5.53 & 7.86 & 296.48 & 11.24 & & \\
\hline & {$\left[\begin{array}{lll}0 & 1 & 0\end{array}\right]$} & 0.31 & 7.27 & 5.97 & 636.46 & 15.19 & & \\
\hline & {$\left[\begin{array}{lll}-1 & 1 & -2\end{array}\right]$} & 0.11 & 5.53 & 7.86 & 296.52 & 11.24 & & \\
\hline & {$\left[\begin{array}{lll}-1 & -1 & -2\end{array}\right]$} & 0.11 & 5.53 & 7.86 & 296.48 & 11.24 & & \\
\hline \multirow[t]{4}{*}{$\left(\begin{array}{lll}1 & 1 & -1\end{array}\right)$} & {$\left[\begin{array}{lll}-1 & 1 & 0\end{array}\right]$} & 0.24 & 7.52 & 5.08 & 409.43 & 14.06 & 4.65 & 1.89 \\
\hline & {$\left[\begin{array}{lll}-1 & -1 & -2\end{array}\right]$} & 0.11 & 4.86 & 7.86 & 302.80 & 9.86 & & \\
\hline & {$\left[\begin{array}{lll}1 & -1 & 0\end{array}\right]$} & 0.24 & 7.52 & 5.08 & 409.43 & 14.06 & & \\
\hline & {$\left[\begin{array}{lll}1 & 1 & 2\end{array}\right]$} & 0.11 & 4.86 & 7.86 & 302.80 & 9.86 & & \\
\hline
\end{tabular}

${ }^{*} \Phi_{P B C}$ is the kink energy in $\mathrm{kcal} / \mathrm{mol} ; a_{P}$ is the propagation length in $\AA$; $a_{E}$ is the growth unit width along each chain in $\AA ; l_{c}$ is the critical length in $\AA ; R$ is the relative growth rate of each face; $S=1.01$ and $T=298 \mathrm{~K}$. 
Table S3: Detailed Results: benzoic acid grown from vapor*

\begin{tabular}{|c|c|c|c|c|c|c|c|c|}
\hline Face & Step edge & $\Phi_{P B C}$ & $a_{P}$ & $a_{E}$ & $l_{c}$ & Step velocity & $d_{h k l}$ & $R$ \\
\hline \multirow[t]{6}{*}{$\left(\begin{array}{lll}0 & 0 & 2\end{array}\right)$} & {$\left[\begin{array}{lll}0 & -1 & 0\end{array}\right]$} & 5.42 & 5.50 & 5.13 & 2391.27 & $2.51 \mathrm{E}-19$ & 10.88 & 1 \\
\hline & {$\left[\begin{array}{lll}-1 & 0 & 0\end{array}\right]$} & 2.57 & 5.13 & 5.50 & 1217.08 & $2.82 \mathrm{E}-17$ & & \\
\hline & {$\left[\begin{array}{lll}-1 & 1 & 0\end{array}\right]$} & 2.27 & 3.75 & 7.52 & 1469.87 & $3.39 \mathrm{E}-17$ & & \\
\hline & {$\left[\begin{array}{lll}0 & 1 & 0\end{array}\right]$} & 5.42 & 5.50 & 5.13 & 2391.27 & $2.51 \mathrm{E}-19$ & & \\
\hline & {$\left[\begin{array}{lll}1 & 0 & 0\end{array}\right]$} & 2.57 & 5.13 & 5.50 & 1217.08 & $2.82 \mathrm{E}-17$ & & \\
\hline & {$\left[\begin{array}{lll}1 & -1 & 0\end{array}\right]$} & 2.27 & 3.75 & 7.52 & 1469.87 & $3.39 \mathrm{E}-17$ & & \\
\hline \multirow[t]{6}{*}{$\left(\begin{array}{lll}1 & 0 & -2\end{array}\right)$} & {$\left[\begin{array}{lll}0 & 1 & 0\end{array}\right]$} & 5.42 & 11.63 & 5.13 & 2391.27 & $5.30 \mathrm{E}-19$ & 5.15 & 1.89 \\
\hline & {$\left[\begin{array}{lll}-2 & 1 & -1\end{array}\right]$} & 0.65 & 5.01 & 11.91 & 666.48 & $5.03 \mathrm{E}-16$ & & \\
\hline & {$\left[\begin{array}{lll}-2 & -1 & -1\end{array}\right]$} & 0.65 & 5.01 & 11.91 & 666.48 & $5.03 \mathrm{E}-16$ & & \\
\hline & {$\left[\begin{array}{lll}0 & -1 & 0\end{array}\right]$} & 5.42 & 11.63 & 5.13 & 2391.27 & $5.30 \mathrm{E}-19$ & & \\
\hline & {$\left[\begin{array}{lll}2 & -1 & 1\end{array}\right]$} & 0.65 & 5.01 & 11.91 & 666.48 & $5.03 \mathrm{E}-16$ & & \\
\hline & {$\left[\begin{array}{lll}2 & 1 & 1\end{array}\right]$} & 0.65 & 5.01 & 11.91 & 666.48 & $5.03 \mathrm{E}-16$ & & \\
\hline \multirow[t]{6}{*}{$\left(\begin{array}{lll}1 & 0 & 0\end{array}\right)$} & {$\left[\begin{array}{lll}0 & -1 & 0\end{array}\right]$} & 5.42 & 10.98 & 5.13 & 2391.27 & $5.00 \mathrm{E}-19$ & 5.45 & 2.42 \\
\hline & {$\left[\begin{array}{lll}0 & -1 & 1\end{array}\right]$} & 0.54 & 4.99 & 11.27 & 524.08 & $5.64 \mathrm{E}-16$ & & \\
\hline & {$\left[\begin{array}{lll}0 & 1 & 1\end{array}\right]$} & 0.54 & 4.99 & 11.27 & 524.08 & $5.64 \mathrm{E}-16$ & & \\
\hline & {$\left[\begin{array}{lll}0 & 1 & 0\end{array}\right]$} & 5.42 & 10.98 & 5.13 & 2391.27 & $5.00 \mathrm{E}-19$ & & \\
\hline & {$\left[\begin{array}{lll}0 & 1 & -1\end{array}\right]$} & 0.54 & 4.99 & 11.27 & 524.08 & $5.64 \mathrm{E}-16$ & & \\
\hline & {$\left[\begin{array}{lll}0 & -1 & -1\end{array}\right]$} & 0.54 & 4.99 & 11.27 & 524.08 & $5.64 \mathrm{E}-16$ & & \\
\hline \multirow[t]{6}{*}{$\left(\begin{array}{lll}0 & 1 & 1\end{array}\right)$} & {$\left[\begin{array}{lll}1 & 0 & 0\end{array}\right]$} & 2.57 & 11.18 & 5.50 & 1217.08 & $6.14 \mathrm{E}-17$ & 4.99 & 183 \\
\hline & {$\left[\begin{array}{lll}2 & -1 & 1\end{array}\right]$} & 0.65 & 5.16 & 11.91 & 666.48 & $5.19 \mathrm{E}-16$ & & \\
\hline & {$\left[\begin{array}{lll}0 & -1 & 1\end{array}\right]$} & 0.54 & 5.46 & 11.27 & 524.08 & $6.16 \mathrm{E}-16$ & & \\
\hline & {$\left[\begin{array}{lll}-1 & 0 & 0\end{array}\right]$} & 2.57 & 11.18 & 5.50 & 1217.08 & $6.14 \mathrm{E}-17$ & & \\
\hline & {$\left[\begin{array}{lll}-2 & 1 & -1\end{array}\right]$} & 0.65 & 5.16 & 11.91 & 666.48 & $5.19 \mathrm{E}-16$ & & \\
\hline & {$\left[\begin{array}{lll}0 & 1 & -1\end{array}\right]$} & 0.54 & 5.46 & 11.27 & 524.08 & $6.16 \mathrm{E}-16$ & & \\
\hline
\end{tabular}

${ }^{*} \Phi_{P B C}$ is the kink energy in $\mathrm{kcal} / \mathrm{mol} ; a_{P}$ is the propagation length in $\AA$; $a_{E}$ is the growth unit width along each chain in $\AA ; l_{c}$ is the critical length in $\AA ; R$ is the relative growth rate of each face; $S=1.04$ and $T=298 \mathrm{~K}$. 
Table S4: Detailed Results: tazofelone form III grown from toluene using the vOCG solvent model*

\begin{tabular}{|c|c|c|c|c|c|c|c|c|}
\hline Face & Step edge & $\Phi_{P B C}$ & $a_{P}$ & $a_{E}$ & $l_{c}$ & Step velocity & $d_{h k l}$ & $R$ \\
\hline \multirow[t]{6}{*}{$\left(\begin{array}{lll}0 & 1 & 1\end{array}\right)$} & {$\left[\begin{array}{lll}1 & 0 & 0\end{array}\right]$} & 9.30 & 8.52 & 11.29 & 9039.93 & 4.24E-58 & 9.50 & 1 \\
\hline & {$\left[\begin{array}{lll}1 & -1 & 1\end{array}\right]$} & 10.71 & 9.40 & 10.24 & 9442.93 & $3.89 \mathrm{E}-59$ & & \\
\hline & {$\left[\begin{array}{lll}-1 & -1 & 1\end{array}\right]$} & 10.62 & 9.43 & 10.21 & 9337.75 & $4.52 \mathrm{E}-59$ & & \\
\hline & {$\left[\begin{array}{lll}-1 & 0 & 0\end{array}\right]$} & 9.30 & 8.52 & 11.29 & 9039.93 & 4.24E-58 & & \\
\hline & {$\left[\begin{array}{lll}-1 & 1 & -1\end{array}\right]$} & 10.71 & 9.40 & 10.24 & 9442.93 & $3.89 \mathrm{E}-59$ & & \\
\hline & {$\left[\begin{array}{lll}1 & 1 & -1\end{array}\right]$} & 10.62 & 9.43 & 10.21 & 9337.75 & $4.52 \mathrm{E}-59$ & & \\
\hline \multirow[t]{4}{*}{$\left(\begin{array}{lll}1 & 1 & 0\end{array}\right)$} & {$\left[\begin{array}{lll}-1 & 1 & -1\end{array}\right]$} & 10.71 & 9.92 & 10.24 & 9442.93 & 4.11E-59 & 9.00 & 2.83 \\
\hline & {$\left[\begin{array}{lll}1 & -1 & -1\end{array}\right]$} & 6.65 & 10.19 & 9.97 & 5704.78 & 4.03E-56 & & \\
\hline & {$\left[\begin{array}{lll}1 & -1 & 1\end{array}\right]$} & 10.71 & 9.92 & 10.24 & 9442.93 & 4.11E-59 & & \\
\hline & {$\left[\begin{array}{lll}-1 & 1 & 1\end{array}\right]$} & 6.65 & 10.19 & 9.97 & 5704.78 & 4.03E-56 & & \\
\hline \multirow[t]{4}{*}{$\left(\begin{array}{lll}1 & 0 & 1\end{array}\right)$} & {$\left[\begin{array}{lll}1 & 1 & -1\end{array}\right]$} & 10.62 & 9.50 & 10.21 & 9337.75 & 4.57E-59 & 9.43 & 3.44 \\
\hline & {$\left[\begin{array}{lll}1 & -1 & -1\end{array}\right]$} & 6.65 & 9.73 & 9.97 & 5704.78 & $3.84 \mathrm{E}-56$ & & \\
\hline & {$\left[\begin{array}{lll}-1 & -1 & 1\end{array}\right]$} & 10.62 & 9.50 & 10.21 & 9337.75 & 4.57E-59 & & \\
\hline & {$\left[\begin{array}{lll}-1 & 1 & 1\end{array}\right]$} & 6.65 & 9.73 & 9.97 & 5704.78 & $3.84 \mathrm{E}-56$ & & \\
\hline
\end{tabular}

* $\Phi_{P B C}$ is the kink energy in $\mathrm{kcal} / \mathrm{mol} ; a_{P}$ is the propagation length in $\AA$; $a_{E}$ is the growth unit width along each chain in $\AA ; l_{c}$ is the critical length in $\AA$; $R$ is the relative growth rate of each face; $S=1.04$ and $T=298 \mathrm{~K}$. 

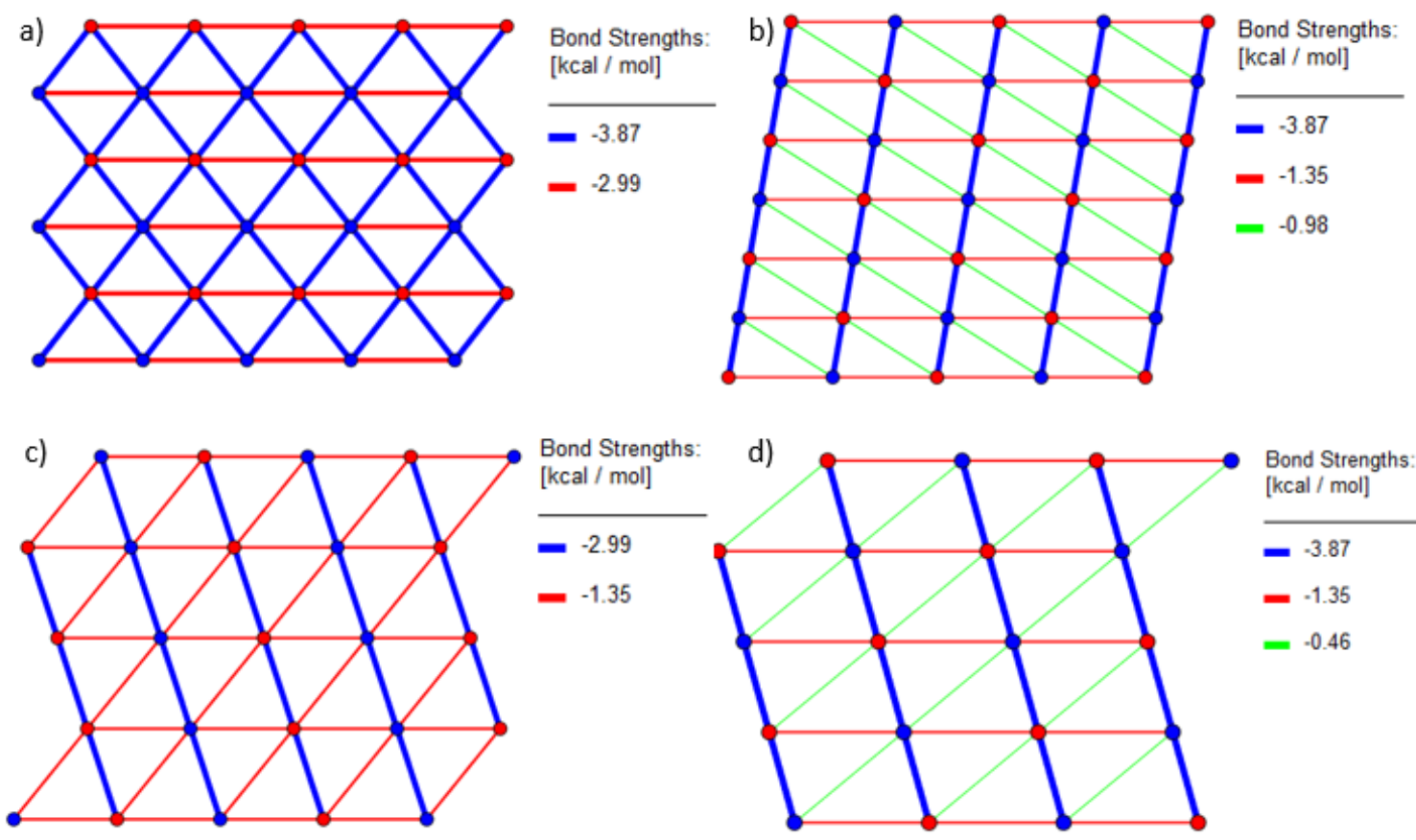

Figure S2. Bonding structures of different F-faces for naphthalene (a) (l0 0 1) face, (b) (1 $1 \square 0)$ face, (c) $(20$-1) face and (d) (1 1 -1) face
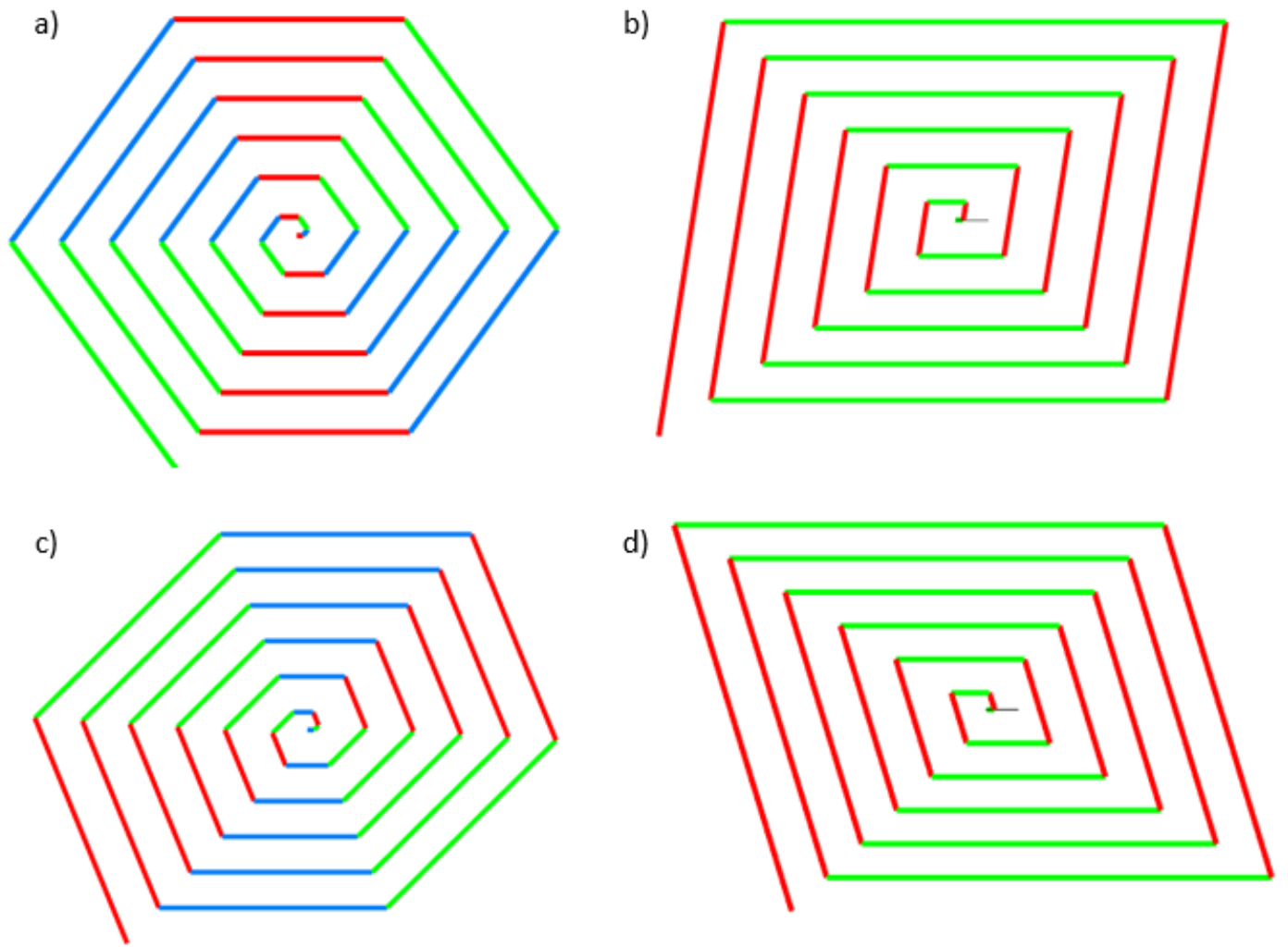

Figure S3. Spiral shapes of different F-faces for naphthalene grown from ethanol (a) $\left(\begin{array}{lll}0 & 0 & 1\end{array}\right)$ face, (b) (1 110$)$ face, (c) (2 0 -1) face and (d) (1 11 -1) face 
a)

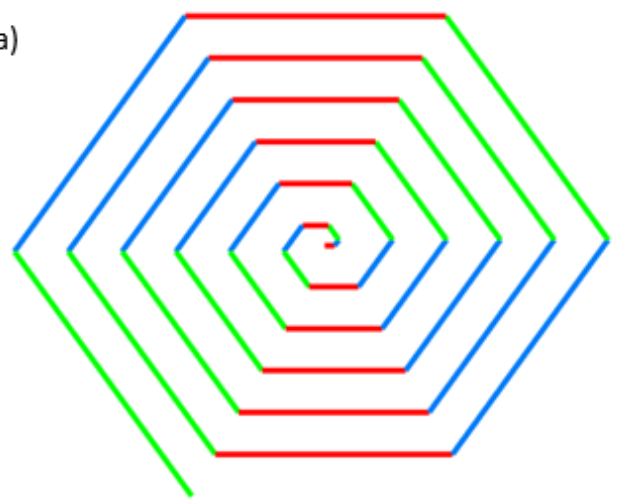

c)

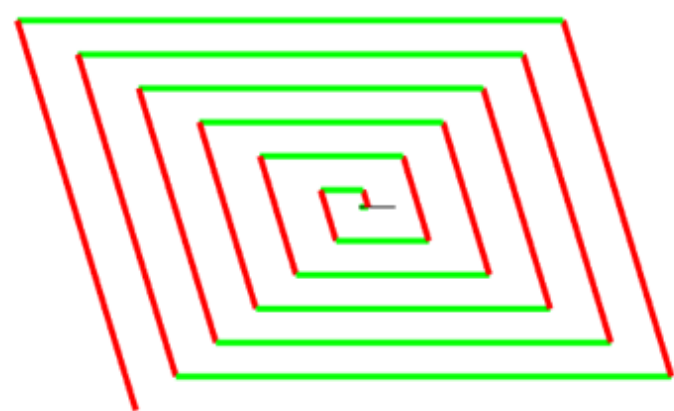

b)

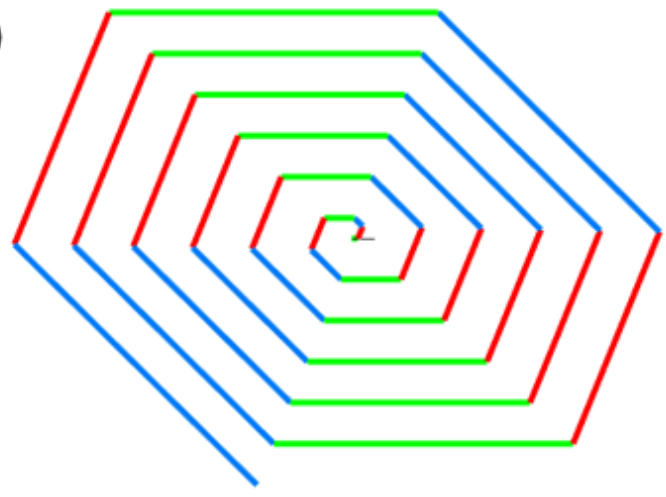

Figure S4. Spiral shapes of different F-faces for naphthalene grown from cyclohexane (a) (0 0 1) face, (b) (2 0 -1) face and (c) (1 1 -1) face

As mentioned in the main body of the paper, the centrosymmetric of benzoic acid dimers growth were used to predict the crystal morphology crystal grown from the vapor phase. As shown in Table S3, the calculated growth rate of the $\left\{\begin{array}{lll}0 & 1 & 1\end{array}\right\}$ face family is about 183 times that of the $\left\{\begin{array}{lll}0 & 0 & 2\end{array}\right\}$ face family $(S=1.04, T=298.00 \mathrm{~K})$. The main reason is that the step velocity values of the different step edges of $\left\{\begin{array}{llll}0 & 1 & 1\end{array}\right\}$ face family are faster that of $\left\{\begin{array}{lll}0 & 0 & 2\end{array}\right\}$ face family, resulting in a faster growth rate of $\left\{\begin{array}{lll}0 & 1 & 1\end{array}\right\}$ face family. Since the benzoic acid dimer is centrosymmetric, the kink rate values of each step edge are equal, so the step velocity values depend mainly on the values of kink density. As shown in Eq. (25), the kink density is mainly determined by the kink energy $\left(\phi_{i}^{K}\right)$. 


$$
\rho_{K, i}=\frac{2 \exp \left(-\frac{\phi_{i}^{K}}{k_{B} T}\right)}{1+2 \exp \left(-\frac{\phi_{i}^{K}}{k_{B} T}\right)}
$$

where $\phi_{i}^{K}$ is kink energy, $T$ is temperature, and $k_{B}$ is Boltzmann constant

For example, as shown in Figure S5 a) and d), the blue PBC's kink energy for $\left.\begin{array}{llll}0 & 0 & 2\end{array}\right\}$ face family is about $5.19 \mathrm{kcal} / \mathrm{mol}$, and blue PBC's kink energy for $\left\{\begin{array}{lll}0 & 1 & 1\end{array}\right\}$ face family is 2.57 $\mathrm{kcal} / \mathrm{mol}$. Thus, according to Eq. (25), the kink density value of the latter is 187 times that of the former, which is very close to 183 (the ratio of face growth rates). Therefore, we can conclude that since the PBCs of the $\left\{\begin{array}{llll}0 & 1 & 1\end{array}\right\}$ face family have lower kink energy values, the growth rate of $\left\{\begin{array}{lll}0 & 1 & 1\end{array}\right\}$ is faster (183 times) than the $\left\{\begin{array}{lll}0 & 0 & 2\end{array}\right\}$ face family, and the crystal shape for benzoic acid should be need-like.

a)

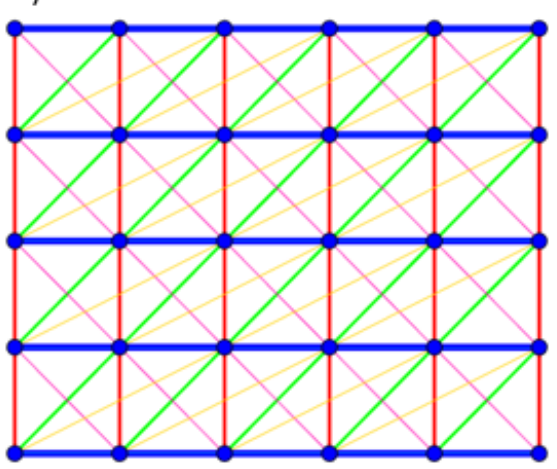

c)

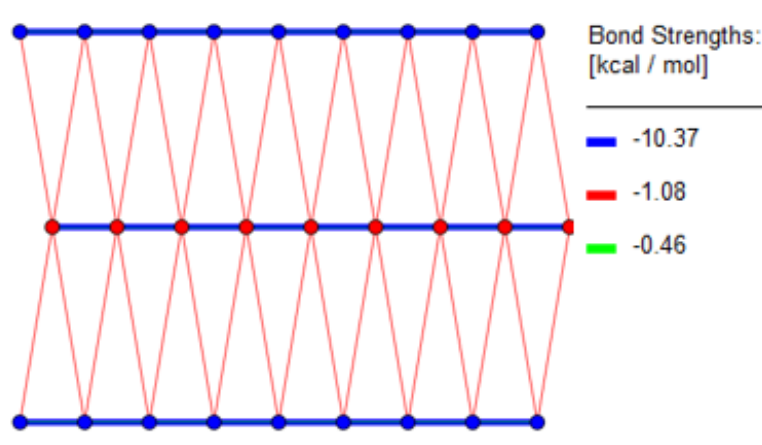

b)
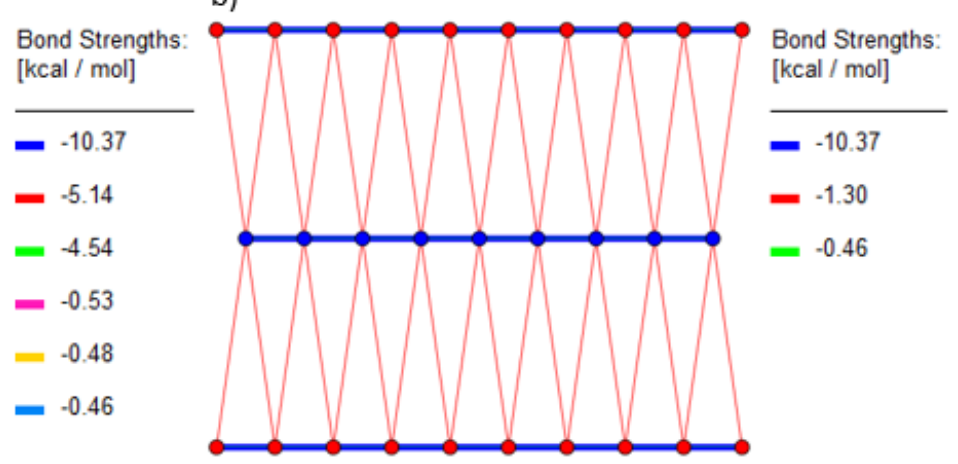

d)

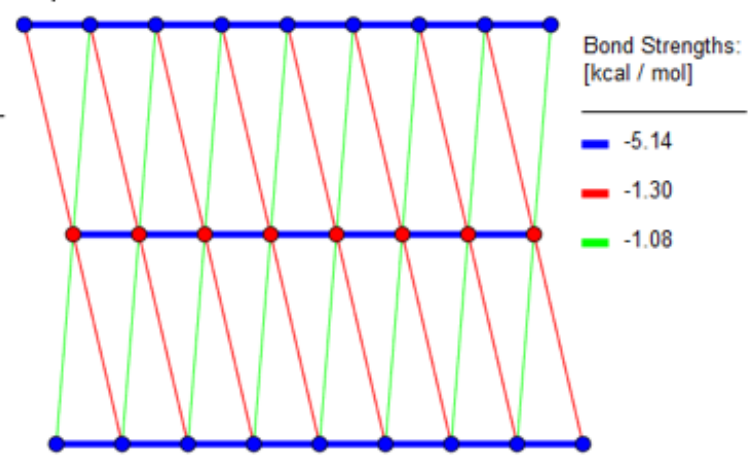

Figure S5. Bonding structures of different F-faces for benzoic acid (a) ( 00 -2) face, (b) (1 0 -2) face, (c) (1 110$)$ face and (d) (llll $\left.\begin{array}{lll}1 & 1\end{array}\right)$ face 
a)

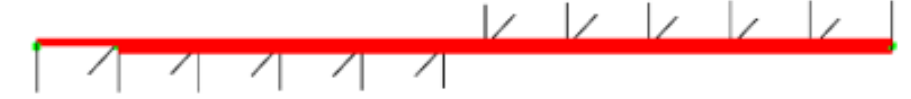

b)

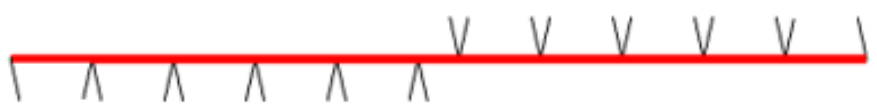

c)

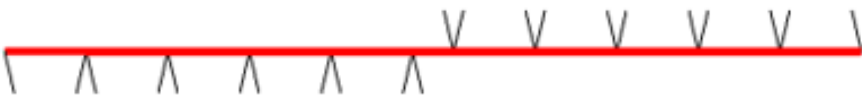

d)

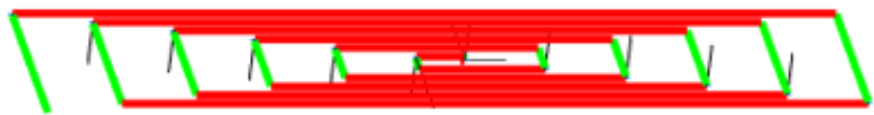

Figure S6. Spiral shapes of different F-faces for benzoic acid grown from vapor (a) $\left(\begin{array}{lll}0 & 0 & -2\end{array}\right)$ face, (b) (1 0 -2) face, (c) (1 110$)$ face and (d) (l $\left.\begin{array}{lll}0 & 1 & 1\end{array}\right)$ face

a)

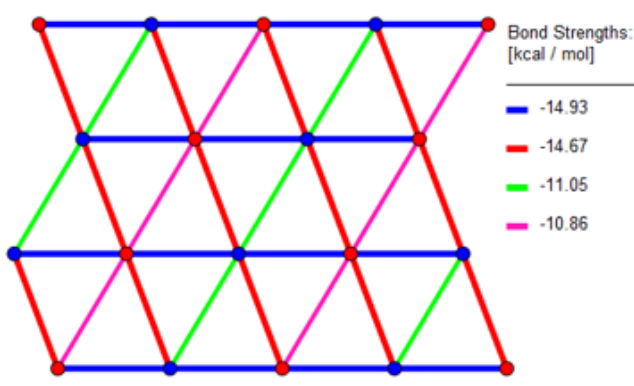

c)

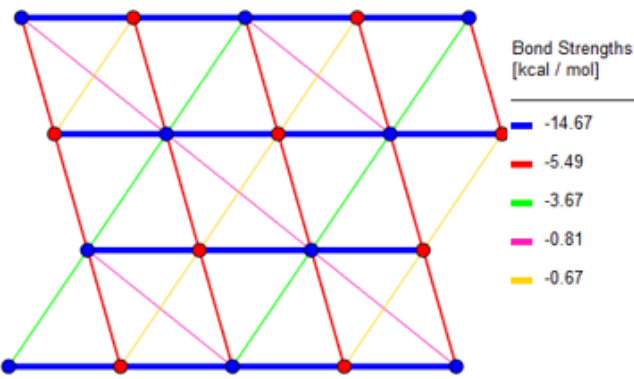

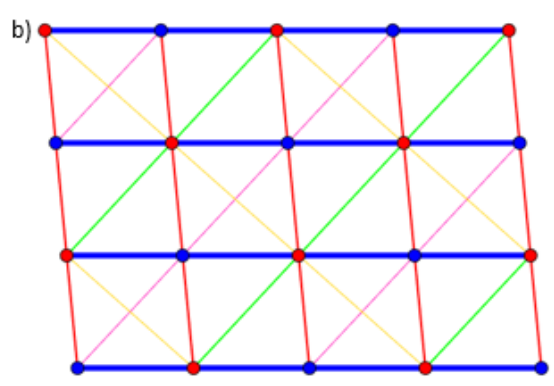

Bond Strengths: [kcal / mol]

- -14.93

$-.49$

$-4.67$

$-0.73$

$-0.45$

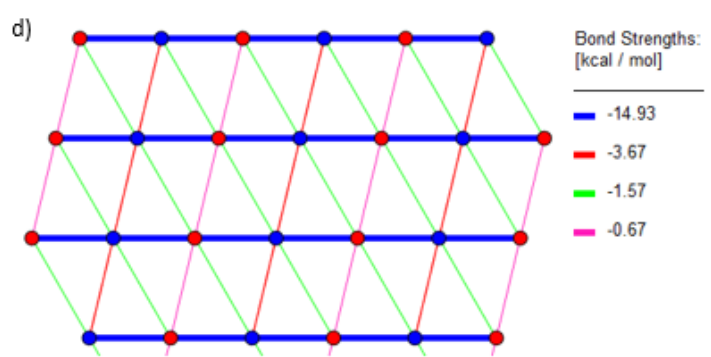

Figure S7. Bonding structures of different F-faces for tazofelone (a) (l $\left.\begin{array}{lll}0 & 1 & 1\end{array}\right)$ face, (b) (llll 1100$)$ face, (c) (1 0 l 1 1) face and (d) (1 0 - -1$)$ face 
a)

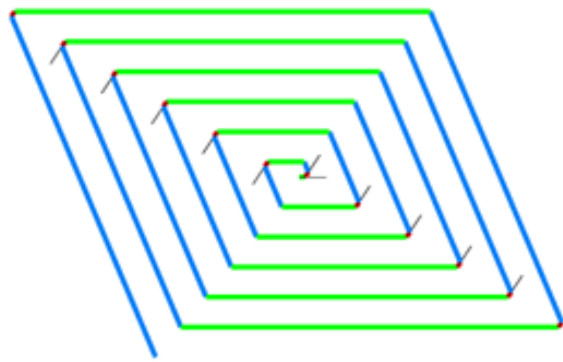

c)

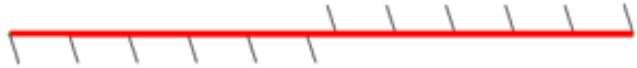

b)

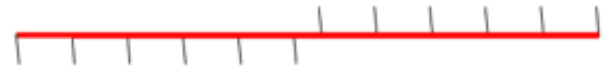

d)

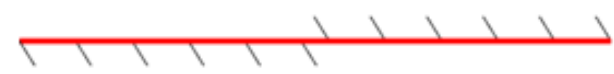

Figure S8. Spiral shapes of different F-faces for tazofelone grown from toluene using the

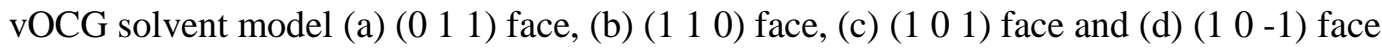

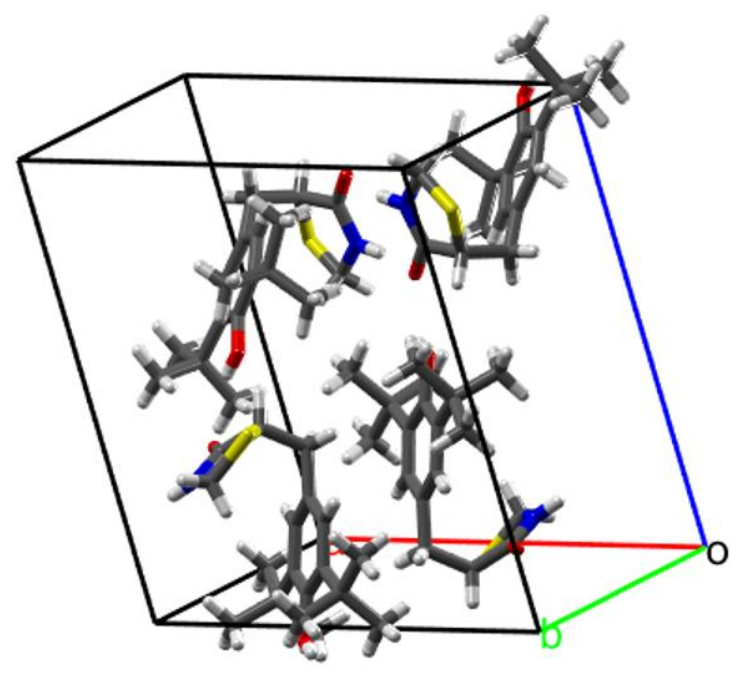

(a) original unit cell (monomer growth unit)

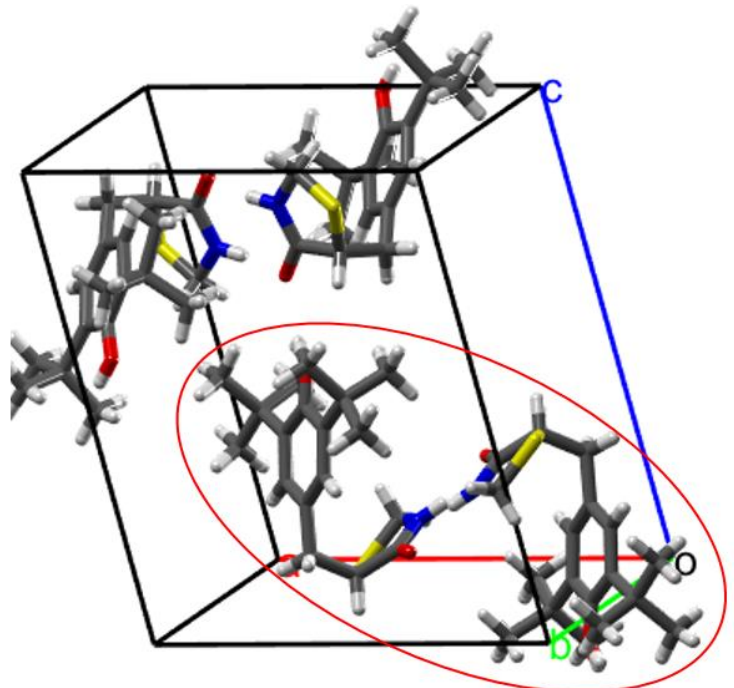

(b) new unit cell (dimer growth unit)

Figure S9. Original unit cell based on four monomer growth units (a) and new unit cell based on two dimer growth units (b) for tazofelone form III, drawing by ADDICT3. The new unit cell has two dimer growth units. For example, the two molecules (monomer growth units) in the red ellipse are one of the dimer growth units. 


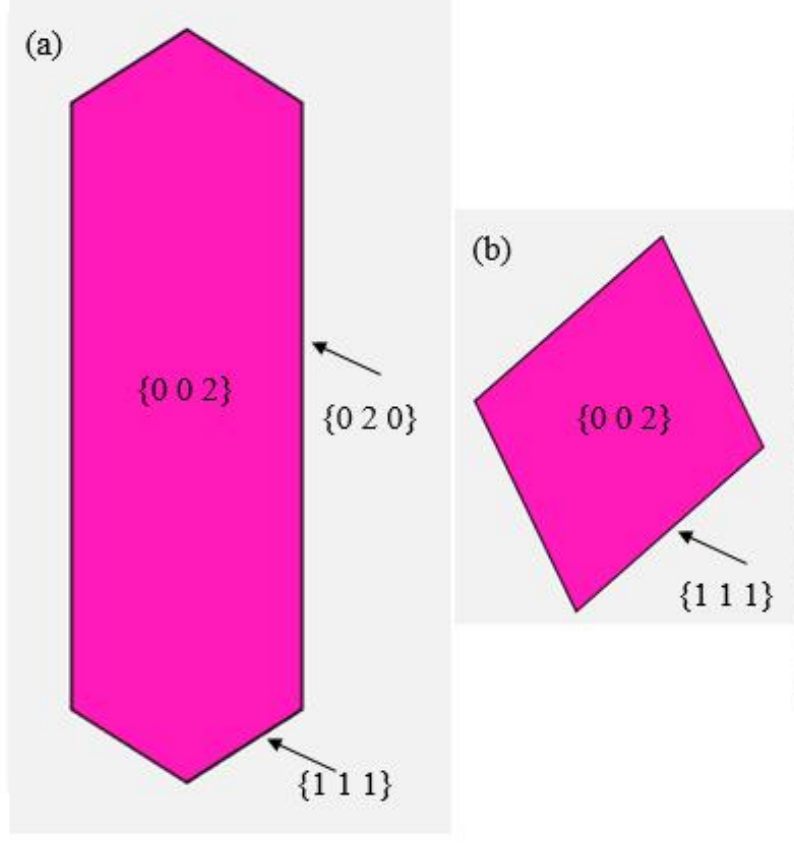

(c)

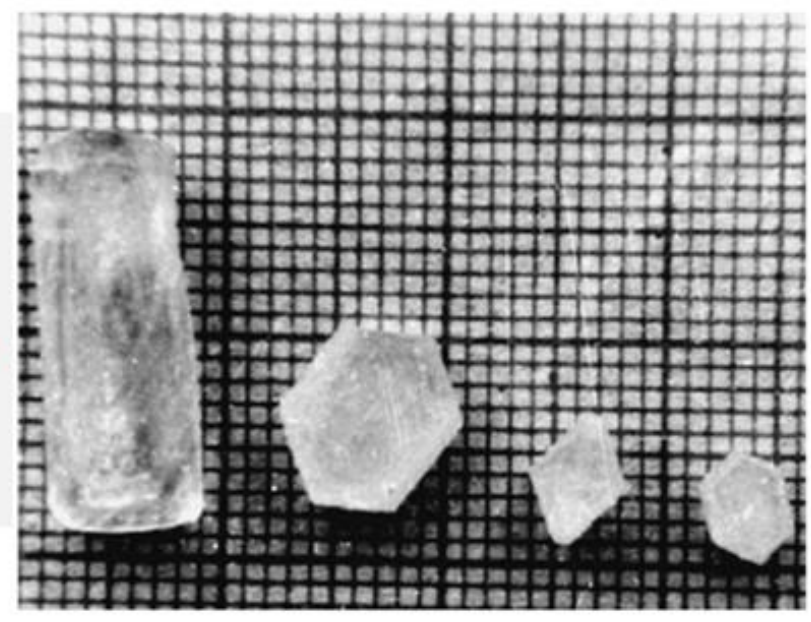

Figure S10. Views of the predicted shape of 4-hydroxyacetophenone ( $S=1.04, T=308 \mathrm{~K}$ ) grown from methanol using the Kaelble solvent model (a) and the vOCG solvent model (b), and the experimental shape grown from methanol (c)*.

*: Chenthamarai, S.; Jayaraman, D.; Meera, K.; Santhanaraghavan, P.; Subramanian, C.; Bocelli, G.; Ramasamy, P. Growth and single crystal XRD characterization of undoped and doped 4-hydroxyacetophenone. Cryst. Eng. 2001, 4, 37-48. 
APPENDIX A

Figure A1 illustrates the diversity of asymmetric growth unit relations within various solid forms. While the asymmetric unit may correspond to one molecule, it may also be a fraction of a molecule or multiple molecules. For organic salts, the molecule may consist of separately charged chemical entities that act as individual growth units. Importantly, the CIF file format contains no information about which atom belongs to which molecule or entity or even how many types exist in the cell. Table A1 provides additional detail for each of the examples in Figure A1, including how one could create the distinct physical growth units starting from the asymmetric unit. Again, this reinforces how starting with the asymmetric unit requires complex, case-by-case treatment. 
a) NAPHTA10; $Z^{\prime}=0.5$

2 growth units / unit cell

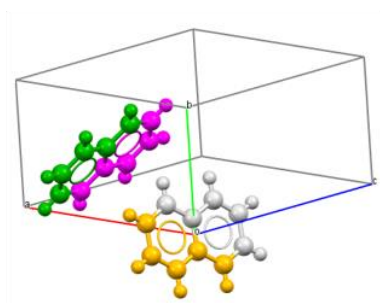

d) AMACET; Z'=1

8 growth units / unit cell

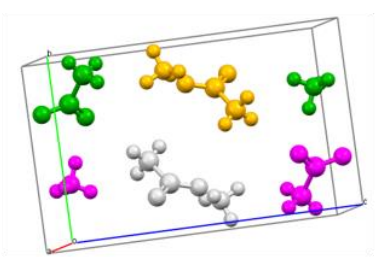

b) ARIFUD; $Z^{\prime}=2$

4 growth units / unit cell

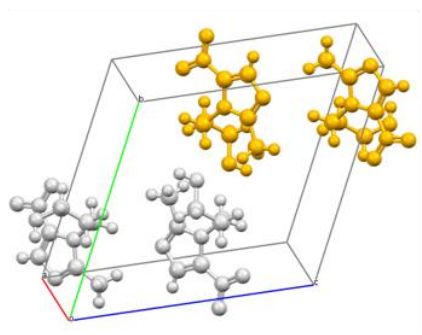

e) BENZACO2; Z'=1

4 growth units / unit cell

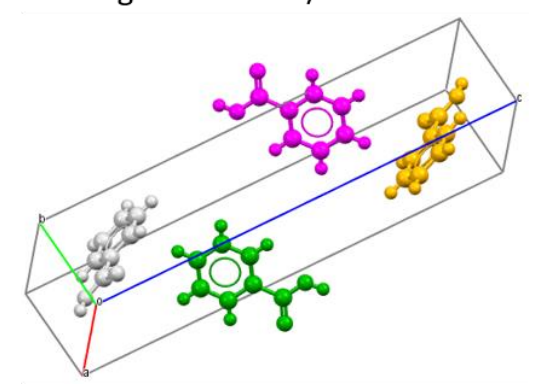

c) HACTPH15; $Z^{\prime}=2$

8 growth units / unit cell

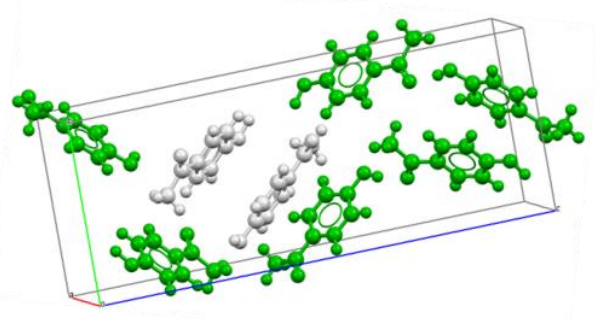

f) FIWZUG; Z'=1

8 growth units / unit cell

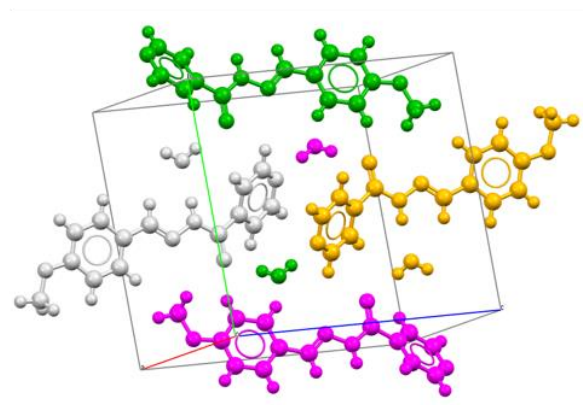

g) PAMQAX; Z'=4

8 growth units / unit cell

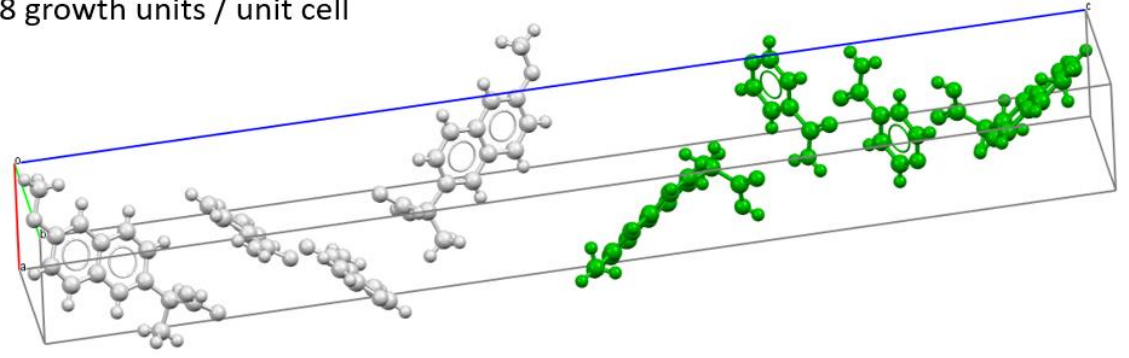

Figure A1. Various crystallographic unit cell examples (CSD codes listed) are coloured by

symmetry operation. The original asymmetric unit appears in a light grey and other

asymmetric units are coloured by their relationship (e.g., inversion symmetry-golden yellow)

to the original asymmetric unit according to the CSD convention. Each separate molecule (or

chemical entity, for the case of salts) acts as a monomeric growth unit, indicating how

producing growth units starting from the asymmetric unit requires different algorithms for

different types of crystallography. $Z^{v}$ is the number of molecules per asymmetric unit. 
Table A1. Connections between molecules, asymmetric units, symmetry operators and growth units for the crystallographic examples in Figure A1. Note that in certain cases, one must be careful to apply the correct symmetry operators (e.g., naphthalene).

\begin{tabular}{|c|c|c|c|c|c|}
\hline CSD Code & $Z^{\prime}$ & $\begin{array}{l}\text { Number of } \\
\text { symmetry } \\
\text { operators }\end{array}$ & $Z$ & $\begin{array}{l}\text { Number of distinct } \\
\text { monomeric growth } \\
\text { units per unit cell }\end{array}$ & $\begin{array}{l}\text { How to produce growth } \\
\text { units from asymmetric unit }\end{array}$ \\
\hline $\begin{array}{l}\text { NAPHTA10 } \\
\text { (Naphthalene) }\end{array}$ & 0.5 & 4 & 2 & 2 & $\begin{array}{l}\text { Apply } 2 \text { symmetry } \\
\text { operators to asymmetric } \\
\text { unit, then apply } 2 \text { symmetry } \\
\begin{array}{l}\text { operators to resulting } \\
\text { molecule }\end{array}\end{array}$ \\
\hline $\begin{array}{l}\text { ARIFUD } \\
\text { (Ternidazole) }\end{array}$ & 2 & 2 & 4 & 4 & $\begin{array}{l}\text { Split asymmetric unit into } 2 \\
\text { molecules, then apply } 2 \\
\text { symmetry operators to each }\end{array}$ \\
\hline $\begin{array}{l}\text { HАCTPH15 } \\
\text { (4-Hydroxyaceto } \\
\text { phenone) }\end{array}$ & 2 & 4 & 8 & 8 & $\begin{array}{l}\text { Split asymmetric unit into } 2 \\
\text { molecules, then apply } 4 \\
\text { symmetry operators to each }\end{array}$ \\
\hline $\begin{array}{l}\text { HXACAN01 } \\
\text { (Paracetamol) }\end{array}$ & 1 & 4 & 4 & 4 & $\begin{array}{llr}\text { Apply } & 4 & \text { symmetry } \\
\text { operators } & \text { to asymmetric } \\
\text { unit } & & \end{array}$ \\
\hline ABAFAL & 2 & 1 & 2 & 2 & $\begin{array}{l}\text { Split asymmetric unit into } 2 \\
\text { molecules }\end{array}$ \\
\hline $\begin{array}{l}\text { AMACET } \\
\text { (Ammonium } \\
\text { acetate) }\end{array}$ & 1 & 4 & 4 & 8 & $\begin{array}{l}\text { Split asymmetric unit into } 2 \\
\text { chemical entities, then } \\
\text { apply } 4 \text { symmetry operators } \\
\text { to each }\end{array}$ \\
\hline $\begin{array}{l}\text { BENZAC02 } \\
\text { (Benzoic acid) }\end{array}$ & 1 & 4 & 4 & 4 & $\begin{array}{l}\text { Apply } 4 \text { symmetry } \\
\text { operators to asymmetric } \\
\text { unit }\end{array}$ \\
\hline FIWZUG & 2 & 4 & 8 & 8 & $\begin{array}{l}\text { Split asymmetric unit into } 2 \\
\text { molecules, then apply } 4 \\
\text { symmetry operators to each }\end{array}$ \\
\hline $\begin{array}{l}\text { PAMQAX } \\
\text { (Naproxen } \\
\text { isonicotinamide) }\end{array}$ & 4 & 2 & 8 & 8 & $\begin{array}{l}\text { Split asymmetric unit into } 4 \\
\text { molecules, then apply } 2 \\
\text { symmetry operators to each }\end{array}$ \\
\hline
\end{tabular}




\section{APPENDIX B}

The following methods are employed to determine whether the growth unit is a monomer or dimer.

(1) Consult reported experimental literature on the crystal

If there are experimental results reporting that the growth unit is a dimer, you can just click the 'dimer' button on the ADDICT panel. Solute molecules that have strong hydrogen bonding groups are likely to incorporate into the crystal as dimers, e.g., carboxylic acids, especially in non-polar or weakly polar solvents (when the solvent is strongly polar like water then it is possible for the solute molecules to hydrogen bond with the solvent instead of with each other). Evidence to look for includes:

(i) The solute exists primarily as dimers in solution (e.g., glycine; Myerson et al. ${ }^{1,2}$ report that in solution glycine diffuses with a "molecular" mass of 1.8 times the mass of a single glycine molecule, thus suggesting that most glycine molecules diffuse as dimers). Such evidence does not guarantee that the crystal growth unit is a dimer (because the dimer could dissociate into monomers at the crystal surface and then incorporate into the crystal as monomers), but it suggests that the growth unit could be a dimer.

(ii) AFM experiments that measure the dimensions of the crystal surface features as being compatible with a dimer growth unit (e.g., the height of a step is twice the height of a monomer, or other such indicators).

(iii) Solid-state NMR experiments that report the growth unit is a dimer.

(2) Determine the growth unit as a monomer or dimer based on the calculations 
(i) Treat the growth unit as a monomer first, run ADDICT.

(ii) Look through the 'strong bonds' array for the monomeric growth unit, and find the strongest interactions between two neighboring molecules. If the absolute value of the bond energy is large, then the growth unit possibly is a dimer.

(iii) Check the geometric structure of the dimer with Mercury to find whether the dimer is reasonable or not. From your chemical intuition, if the geometric structure is reasonable, run ADDICT with the growth unit as a dimer.

(iv) If we can find the experimental shape, compare the predicted crystal shapes (with growth units both as a monomer and dimer).

If the predicted crystal shape with monomeric growth unit agrees with the experimental shape, then the growth unit for this crystal is a monomer. Alternatively, if the predicted crystal shape with dimeric growth unit agrees with the experimental shape, then the growth unit for this crystal is a dimer.

\section{APPENDIX C}

In this section, we take naphthalene as an example to demonstrate how to appropriately interpret the crystallography step-by-step according to the main flow in Figure 3.

\section{- Input CIF: any format, any $Z^{\prime}$}

The cif file for naphthalene can be found in CCDC database or Mercury software. Its database identifier is NAPHTA10. The input file to ADDICT is NAPHTA10.cif.

\section{- Open in Mercury, pack unit cell, export as MOL2 format}

When using Mercury to open the NAPHTA10.cif input file, Figure C1 can be displayed in the main interface of Mercury. There is only one naphthalene molecule containing 18 atoms; half 
belong to the asymmetric unit (light grey), the other half are generated using an inversion symmetry operation (golden yellow). Then, click "packing" button in Mercury and Figure C2 can be obtained. The packed naphthalene unit cell contains a total of 10 molecules, of which 8 are at the apex of the unit cell and 2 at the center of faces. Save this packed unit cell as NAPHTA10.MOL2 file.

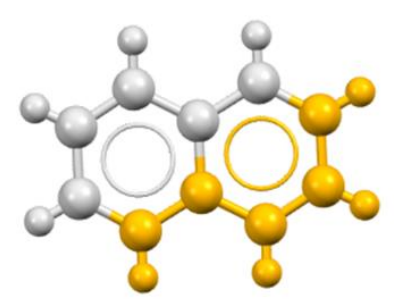

Figure C1. Naphthalene molecule is coloured by symmetry operation.

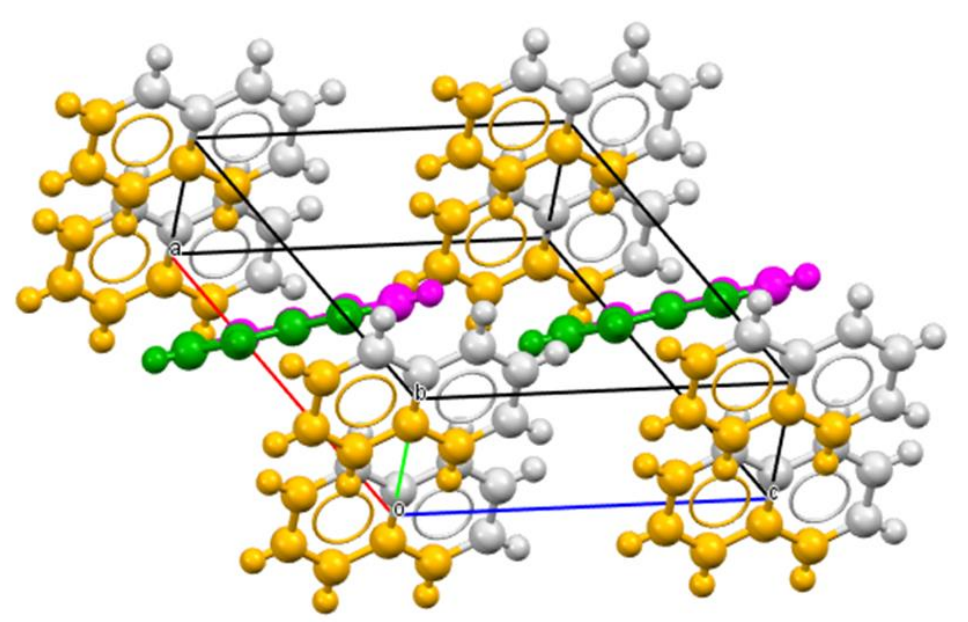

Figure C2. Packed unit cell for Naphthalene crystal coloured by symmetry operations.

- Read MOL2 Atoms: type, orthonormal coordinates and which molecule they belong to

ADDICT can read each atom in the above NAPHTA10.MOL2 file including the atom type, orthonormal coordinates, and which molecule they belong to. In this case, there are a total of 180 atoms, and every 18 atoms belongs to one molecule, that is, a total of 10 molecules are read in and identified. 
- Calculate molecular centers of mass, eliminate repeated growth units (unit cell surface)

Based on the information read from the MOL2 file, ADDICT can then calculate molecular centers of mass. According to these centers of mass, repeated growth units can be removed. As shown in Figure C3, only two naphthalene molecules, which have a total of four asymmetric units remain in the unit cell.

- Have now obtained each type of monomeric growth unit in the unit cell

At this point, ADDICT identifies two naphthalene growth units and outputs files that the Gaussian software can recognize.

- Run Gaussian + Antechamber to compute atomic partial charges.

ADDICT receives partial charges for each naphthalene growth unit based on Gaussian and Antechamber calculations.

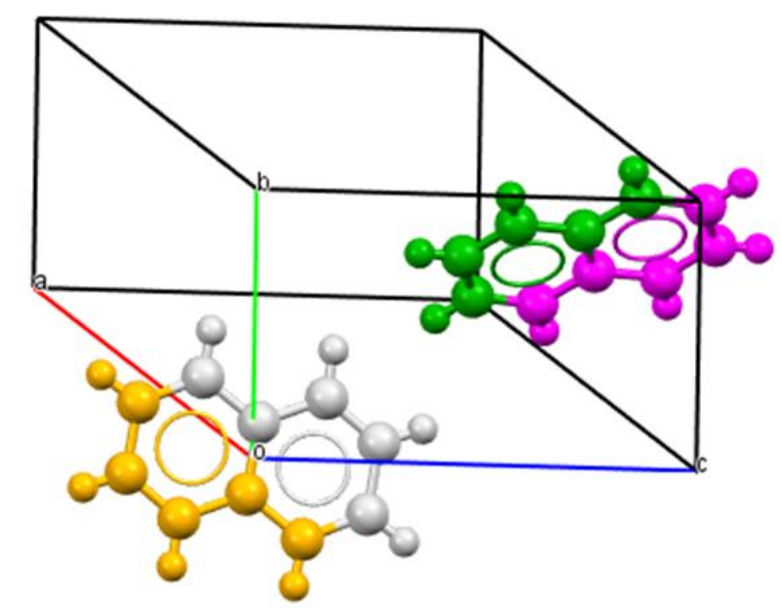

Figure C3. Naphthalene unit cell after eliminating repeated growth units (coloured by Symmetry operation). 


\section{References}

(1) Chang, Y. C.; Mayerson, A. S. Diffusivity of glycine in concentrated saturated and supersaturated aqueous solutions. AIChE J. 1986, 32, 1567-1569.

(2) Myerson, A. S.; Lo, P. Y. Diffusion and cluster formation in supersaturated solutions. J. Cryst. Growth 1990, 99, 1048-1052. 